\title{
Deep Prior Based Unsupervised Reconstruction of Irregularly Sampled Seismic Data
}

\author{
Fantong Kong, Francesco Picetti,Vincenzo Lipari, Paolo Bestagini, Member, IEEE, Xiaoming Tang \\ and Stefano Tubaro, Senior Member, IEEE
}

\begin{abstract}
Irregularity and coarse spatial sampling of seismic data strongly affect the performances of processing and imaging algorithms. Therefore, interpolation is a usual pre-processing step in most of the processing workflows. In this work, we propose a seismic data interpolation method based on the deep prior paradigm: an ad-hoc Convolutional Neural Network is used as a prior to solve the interpolation inverse problem, avoiding any costly and prone-to-overfitting training stage. In particular, the proposed method leverages a multi resolution U-Net with 3D convolution kernels exploiting correlations in cubes of seismic data, at different scales in all directions. Numerical examples on different corrupted synthetic and field datasets show the effectiveness and promising features of the proposed approach.
\end{abstract}

Index Terms-Seismic data processing, Interpolation, Inverse problems, Convolutional Neural Networks.

\section{INTRODUCTION}

$\mathbf{E}$ CONOMIC or environmental constraints, cable feathering in marine acquisitions and the presence of dead or damaged traces result in most seismic datasets being poorly and irregularly sampled in space. As a result, the vast majority of seismic processing and imaging workflows [1] -[3] typically include a preliminary interpolation step. The importance of the issue is confirmed by the vast number of methods proposed and the vitality of the relevant scientific literature.

Interpolation of seismic data is an ill-posed inverse problem. Therefore, some reasonable a-priori information must be used in order to solve it, at least implicitly. Depending on the underlying hypothesis, interpolation methods can be roughly grouped in four families.

Model driven techniques make simplifying assumptions on subsurface velocities and implicitly apply a migrationdemigration pair [4], [5]. The performance of these techniques is strongly hindered by complex structural burden.

A second family of approaches models seismic data as a (local) superposition of plane waves and leverages based on prediction filters. These techniques mainly target regularly sampled data [6].

A third alternative relies on the fact that seismic data are inherently low-rank due to their repetitive features [7], whereas

F. Kong and X. Tang are with the School of Geosciences, China University of Petroleum (East), 266580, Qingdao, China (e-mail: kft_upc@ @otmail.com).

F. Picetti, V. Lipari, P. Bestagini and S. Tubaro are with the Dipartimento di Elettronica, Informazione e Bioingegneria, Politecnico di Milano, 20133 Milan, Italy (e-mail: vincenzo.lipari@polimi.it).

The authors gratefully acknowledge financial support from China Scholarship Council and Fundamental Research Funds for the Central Universities (grant 18CX06021A). GPU computation has been made available thanks to the GPU Grant Program by NVIDIA Corporation, to which the authors express deep gratitude. missing traces increase their rank. These methods recast interpolation problems as rank reduction and matrix/tensor completion ones [8]-[10].

Finally, a fourth family of interpolation algorithms leverages the hypothesis that seismic data are characterized by a parsimonious representation in some transform domain. Data can be well represented by only a few non-zero transform coefficients; therefore, they can be recovered by imposing some sparsity constraint [11]-[14]. These methods assume that it is possible to represent the data as a linear combination of atoms (i.e., elementary signals) taken from a dictionary (i.e., a fixed set of atoms). However, a fixed basis defines only a subset of the transforms and alternatively represents clean signals as a linear combination, under a sparsity constraint, of the atoms in a learned overcomplete dictionary [15].

More recently, besides these traditional processing methods, many seismic interpolation methods based on Convolutional Neural Networks (CNNs) have been proposed [16]-[21]. CNNs learn how to reconstruct missing traces during a training step, usually performed on a set of pairs of corresponding corrupted and uncorrupted data. Then, at test time, they are employed to interpolate new data. The performances of these methods strongly depend on training data. However, collecting high-quality and well-structured training datasets is a timeconsuming and expensive operation. In addition, while these techniques have demonstrated cutting-edge performance when training and test data are strongly correlated, generalizing results to different data distributions is difficult.

A different approach has been proposed interpreting the $\mathrm{CNN}$ architecture as a deep prior in the framework of inverse problems to address tasks such as inpainting, denoising or super-resolution [22]. Through this approach, a CNN learns how to map a random noise realization into the solution of the assigned task, only based on the data itself, without the need of a different training dataset. This strategy has been recently explored also for the task of 2D seismic data interpolation using common U-Net as deep prior [23], [24].

Inspired by our previous studies [24], we propose a 3D interpolation method belonging to the deep prior family. A key element in this framework is the specific design of the actual network architecture, which is also the main goal of this letter. More specifically, the contribution of this study can be summarized as follows:

- We analyze the deep prior paradigm used in the context of seismic data interpolation, highlighting some improvement possibilities. 
- Based on these analyses, we propose the MultiResUNet as a suitable deep prior for the task of seismic data interpolation instead of the most commonly used U-Net.

- We make use of 3D convolutional layers to fully exploit correlations in both horizontal and depth/time directions, in contrast to classically used 2D convolutions.

- We propose an inizialization of the CNN weights which greatly accelerate the convergence of the method.

- We test the proposed approach on synthetic and field data, validating it against both a deep prior method based on $\mathrm{U}-\mathrm{Net}$ and more traditional interpolation techniques.

\section{INTERPOLATION VIA DEEP PRIOR}

Let us describe our goal for seismic data interpolation in the framework of inverse problems. We consider an ideal densely and regularly sampled three-dimensional cube of seismic data (e.g., a 3D common shot gather, 2D dataset, a 3D post-stack volume, etc.). This data cube represents the true model we aim at estimating through interpolation. Without loss of generality we can represent such ideal data as a vector $\mathbf{m}_{\text {true }}$. We can think the observed seismic data $\mathbf{d}_{\mathrm{obs}}$ as generated by a linear sampling operator $\mathbf{S}$ applied to $\mathbf{m}_{\text {true }}$ (i.e., a diagonal operator with zeros on the missing data locations and ones elsewhere). Therefore, we can recast the interpolation problem as the inversion of

$$
\mathbf{d}_{\mathrm{obs}}=\mathbf{S m}_{\text {true }}
$$

This is an ill-posed inverse problem: each solution preserving the observed data honours (1). In order to obtain a reasonable solution it is necessary to constrain the inversion by adding some kind of a-priori information. A standard way consists in formulating the inverse problem as finding the solution $\mathbf{m}^{*}$ that minimizes an objective function

$$
J(\mathbf{m})=E\left(\mathbf{S m}-\mathbf{d}_{\mathrm{obs}}\right)+R(\mathbf{m}),
$$

where $E(\cdot)$ is the data fidelity term measuring some distance between modeled and observed data, and $R(\cdot)$ is a regularizer function formalizing the distance of the solution from the space of solutions honouring the desired prior. The choice of the prior $R(\cdot)$ is critical and usually derives from insights of human experts.

Deep Image Prior (DIP) is an alternative solution for the regularization of inverse problems originally proposed in the context of image restoration [22]. Here, a convolutional autoencoder acts as a parametric non linear function $f_{\boldsymbol{\theta}}(\cdot)$ and the problem is recast as finding the set of parameters $\boldsymbol{\theta}^{*}$ which minimizes the objective function

$$
J(\boldsymbol{\theta})=E\left(\mathbf{S} f_{\boldsymbol{\theta}}(\mathbf{z})-\mathbf{d}_{\mathrm{obs}}\right),
$$

where $\boldsymbol{\theta}$ is the set of weights of the CNN and $\mathbf{z}$ is a random noise realization. Through this procedure, rather than minimizing the objective function in the space of the model, we perform the search in the space of the CNN parameters. In other words, the parametrization through a convolutional autoencoder modifies the shape of the objective function, similarly to a preconditioner, and drives the iterative solver toward solutions consistent with the inherent self similarities of seismic data.
Although the optimization goal constrains the CNN output only to fit the observed data $\mathbf{d}_{o b s}$, as a byproduct the missing trace also are adequately reconstructed, because they share the same inner structure captured by the $\mathrm{CNN}$ during the optimization. Therefore, the interpolated solution is obtained as

$$
\mathbf{m}^{*}=f_{\boldsymbol{\theta}^{*}}(\mathbf{z}) .
$$

Notice that, even though the interpolated result is the output of a convolutional neural network, this method does not exploit the deep learning paradigm where a training phase is performed over a specifically designed set of data.

In particular, with the DIP paradigm, only corrupted data are used in the reconstruction process and the CNN implicitly assumes the role of prior information that exploits correlations in the data to learn their inner structure. Therefore, the choice of a specific CNN architecture is critical for a suitable and well-performing solution.

\section{Proposed Network ARChiteCture}

The U-Net is a convolutional autoencoder (i.e., a CNN aiming at reconstructing a processed version of its input) characterized by the so called skip-connections and originally introduced for medical image processing [25]. If properly trained according to the standard deep learning paradigm, it proves very effective for multidimensional signal processing tasks such as denoising, segmentation, inpainting, etc.

More recently, the MultiResUNet [26] has been proposed to improve the performance of the U-Net for multimodal medical image segmentation, based on the consideration that the targets of interest have different shapes and scales. If we want to exploit self-similarities of seismic data, working at different scales can be strongly beneficial. Therefore, we propose an ad-hoc MultiResUNet as a proper deep prior for seismic data interpolation. Moreover, in order to exploit correlations in both horizontal and vertical directions, in the layers of the proposed network we use $3 \mathrm{D}$ convolutions.

In short, the features of the proposed architecture (Fig. 1) can be summarized as follows.

i. All filtering operations in network are fully 3D.

ii. Convolutional layers are replaced by so called Multi Resolution (MultiRes) blocks shown in Fig. 1. This block approximates multi-scale features of the Inception block while limiting the number of parameters of the network, which is critical when employing it as a deep prior.

iii. Skip connections, which are the distinctive feature of the U-Net, are replaced by Residual Path blocks shown in Fig. 1. $\mathcal{E}$ is the output of an encoding layer, and $\mathcal{D}$ is concatenated to the corresponding decoding layer.

iv. Downsampling is achieved by $3 \times 3 \times 3$ convolutions with stride $2 \times 2 \times 2$. Upsampling is performed by nearest interpolation. Batch Normalization and LeakyReLU activation function follow every convolutions apart from the last one (responsible for the CNN output).

By using this MultiResUNet architecture as a deep prior we follow the workflow below for both irregular or regular seismic data interpolation. 


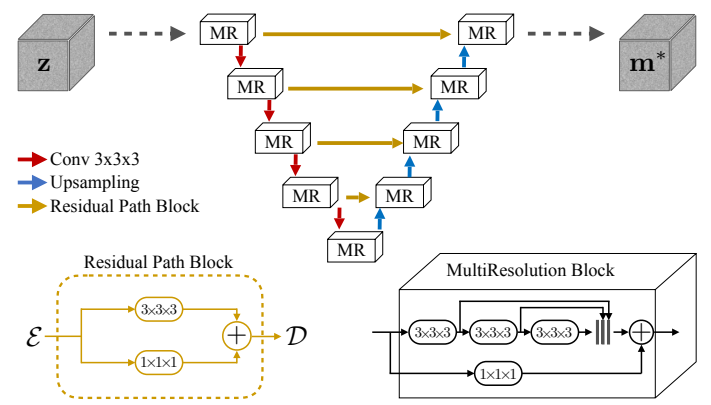

Fig. 1. The MultiResUNet: a Unet-like composed of multi-resolution blocks (bottom right) and linked by residual path blocks (bottom left).

i. We normalize $\mathbf{d}_{\mathrm{obs}}$ by applying a gain function to correct for the geometrical spreading and make data dynamics more suitable for the CNN. In particular, for the test we performed, we imposed the standard deviation of the data to be about 5 after applying the gain function.

ii. The CNN input $\mathbf{z}$ is a volume of white gaussian noise with zero mean and standard deviation of 0.1 .

iii. We optimize the weights of the CNN through the ADAM algorithm by minimizing the $\ell_{1}$ distance between the normalized data $\mathbf{d}_{\mathrm{obs}}$ and the corresponding traces returned by the CNN. At each iteration we perturb the CNN input noise with additional gaussian noise to strengthen the convergence.

iv. After the optimization, the desired interpolated data are obtained by de-normalizing the $\mathrm{CNN}$ output volume to account for $\mathbf{d}_{\mathrm{obs}}$ normalization.

The process of reconstruction of a seismic gather is illustrated in Fig. 2. In particular, it shows a vertical section and a time section of the CNN output at different iterations. First, some blurry structures are recovered; then all the events are gradually reconstructed as the number of iterations increases.

As the DIP based inversion requires a high number of iterations to converge, in order to exploit the proposed method in production applications, it is mandatory to deal with the issues related to its computational cost. We faced this aspect through a strategy similar to transfer learning: contrary to the original DIP algorithm, where the CNN weights are randomly initialized, we start with a pre-trained network. In particular, we observed that convergence is greatly accelerated if we start with the weights computed for nearby data.

Moreover, with regard to computational aspect, it is worth considering that the proposed method can also be applied in a patchwise fashion and its parallelization is straightforward.

\section{EXAMPLES}

In this section we report some examples designed to highlight relevant features of the proposed interpolation strategy. In the examples, we used the ADAM optimization algorithm setting the learning rate to 0.001 . For the sake of reproducibility, we provide the codes to replicate these examples in the repository https://github.com/polimi-ispl/deep_prior_interpolation.

Metrics. In order to evaluate the interpolation performance we report two metrics: signal-to-noise ratio (SNR) between $\mathbf{m}_{\text {true }}$ and $\mathbf{m}^{*}$; Pearson correlation coefficient $\gamma$ computed between $\mathbf{m}_{\text {true }}$ and $\mathbf{m}^{*}$, considering only the corrupted samples. Notice that, as these objective metrics do not completely describe the goodness of an interpolation result, we also rely on visual inspection.

3D simple synthetic data. A first set of examples we show is the reconstruction of a simple 3D shot gather including several hyperbolic reflection events and 4 superimposed weak events simulating diffractions (Fig. 3a. The data includes $128 \times 128$ traces of 512 time samples with 50 spatial sampling and $4 \mathrm{~ms}$ time step. We build the corrupted dataset by randomly deleting $66 \%$ of the traces (Fig. $3 \mathrm{~b}$ ).

The corresponding final interpolated section is shown in Fig. $3 \mathrm{c}$. The interpolation result is visibly satisfactory and the SNR achieved is $15.22 \mathrm{~dB}$. The correlation coefficient between the predicted data and the true data is 0.985 .

As a matter of fact, this example has been designed to test the effectiveness of the proposed interpolation on a challenging case with weak events. From the results displayed in Fig. 3 we observe that, despite they are barely visible, the weak events crossing the much more energetic hyperbolas are reconstructed by interpolation.

The wiggle plot in Fig. 4 emphasizes the effectiveness of the achieved result by displaying the comparison between four reconstructed and original traces on the large gap after trace 35. Red filled wiggles indicate the interpolated traces, well fitting the original ones in black.

The effectiveness of the strategy we propose in order to accelerate the convergence is shown in Fig. 5. Here the red continuous and dashed lines display the value of SNR and objective function vs. the iteration number when the CNN weights have been randomly initialized. The blue lines show the corresponding curves for a $\mathrm{CNN}$ with the starting weights set as those learnt after interpolating a nearby gather. Notice that the blue line is always above the red line. Moreover, the pre-trained optimization convergences after 100 iterations approximately, while when starting from scratch it needs more than 2000 iterations to get similar results in terms of both SNR and residual value. This means that, while on a single TITAN V GPU the computation time is $\sim 70$ minutes for 2000 iterations when starting from scratch, the transfer learning strategy allows to obtain a suitable result in $\sim 4$ minutes.

2D synthetic data. The second set of experiments aims at demonstrating the impact of the architecture design and the improvements achieved through 3D convolutions. It should

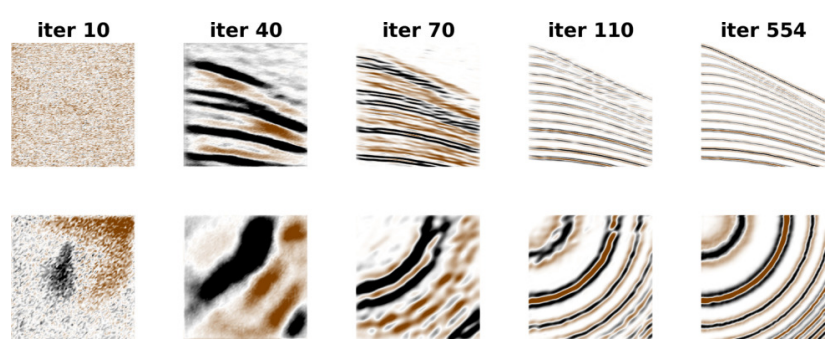

Fig. 2. Reconstruction of seismic data at increasing iterations. 


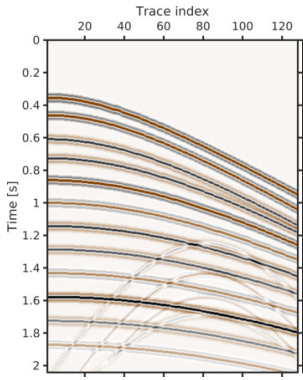

(a)

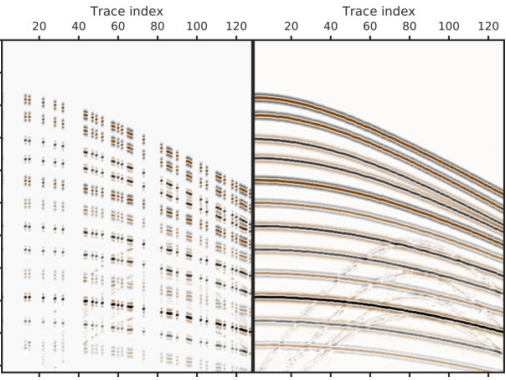

(b)

(c)
Fig. 3. 3D synthetic shot gather with weak events(a): interpolation results of DIP method (c) from irregular sampled data(b).

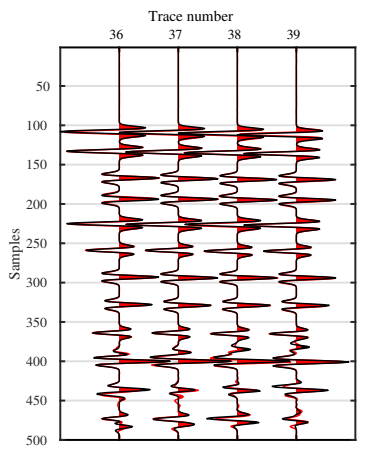

(a)

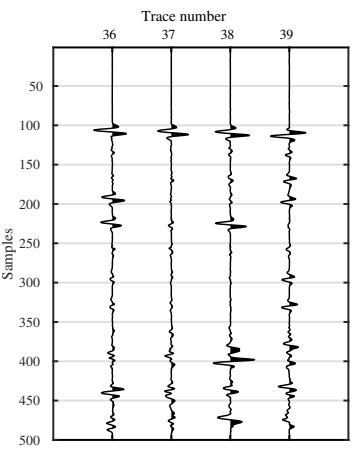

(b)
Fig. 4. (a) Comparison of single traces between original (black line) and interpolated (red wiggles) data. (b) Difference between original and interpolated data plotted in the same scale of Fig. 4a.

be noted that a $2 \mathrm{D}$ seismic dataset is actually a cube whose dimensions are source position, receiver position and time. On the central part of the well known 2D Marmousi model, data are modeled through finite differences on an acquisition geometry of 256 sources and 256 receivers; the source is a Ricker wavelet centered at $15 \mathrm{~Hz}$; the temporal sampling is $8 \mathrm{~ms}$. From this dataset (which is the ground truth of the experiment) we regularly erase $50 \%$ of the traces.

Figs. 6a and 6b show an interpolated shot gather obtained through a standard U-Net deep prior and the corresponding residual, respectively. While the result is visually satisfactory, significant signal leakage is evident in the residual. The SNR in this case is $12.2 \mathrm{~dB}$, and the correlation coefficient between the interpolated data and the ground truth is 0.969 .

By using as a deep prior the MultiResUNet with 2D convolutions we achieved the result shown in Fig. 6c. The corresponding residual, displayed in Fig. 6d, is clearly less energetic but still contaminated by coherent signal leakage

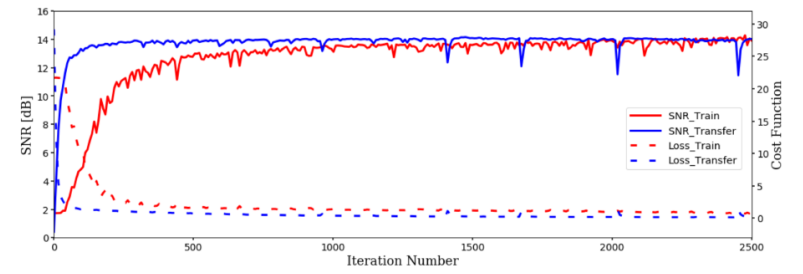

Fig. 5. Cost function and SNR vs. iteration number for the case of initialization from scratch and from the nearby gather respectively.

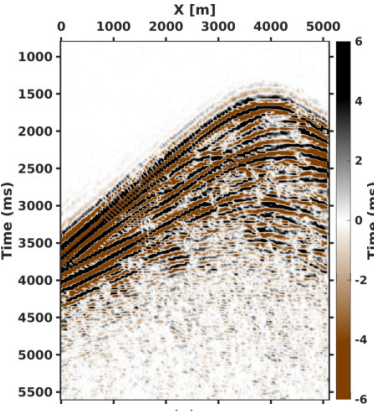

(a)
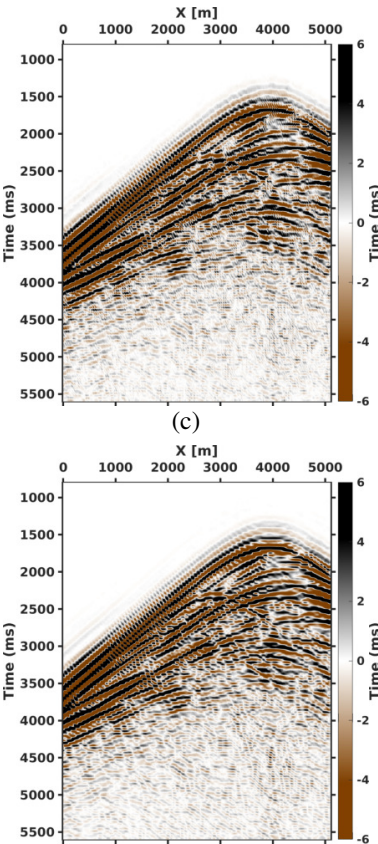

(e)

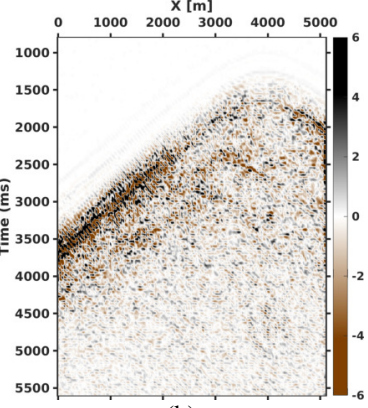

(b)

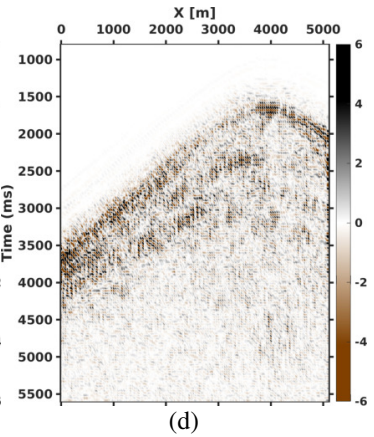

(d)

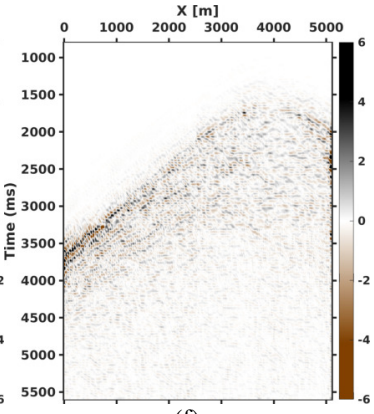

(f)
Fig. 6. Interpolation result and residual of (a)(b) ordinary U-net, (c)(d) 2D MultiResUNet and (e)(f) 3D MultiResUNet, respectively.

throughout the whole gather. The SNR and correlation coefficient is $14.3 \mathrm{~dB}$ and 0.981 , respectively.

Finally, Figs. 6e and 6f depict the output of the proposed MultiResUNet with 3D convolutions and the corresponding residual, respectively. By taking advantage of the correlation and self-similarities between different gathers we are able to greatly limit the residual power and the signal leakage. This improved result is confirmed by the SNR of $22.1 \mathrm{~dB}$. More important, the correlation coefficient between original and restored traces approaches to 0.997 , indicating a good performance.

3D field data. Finally, we test the proposed interpolation strategy on a 3D migrated field data. The migrated volume we use is a portion of 128 inlines (250-378) and crosslines (750878) with a time sampling of $16 \mathrm{~ms}$ from the F3 Netherlands survey [27]. An inline section of the original volume is shown in Fig. $7 \mathrm{a}$ and the corresponding decimated image $(50 \%$ missing traces) is shown in Fig. 7b. For this section is mainly characterized by horizontal and slightly dipping events, which is favorable for rank-reduction methods; we test the proposed 
technique against the multichannel singular-spectrum analysis (MSSA) [8]. The deep prior result in Fig. 7c and the MSSA result in Fig. 7d are visually comparable while SNR (10.36 $\mathrm{dB}$ vs. $7.81 \mathrm{~dB}$ ) and correlation coefficient (0.935 vs. 0.910$)$ indicate a better performance of the deep prior method.

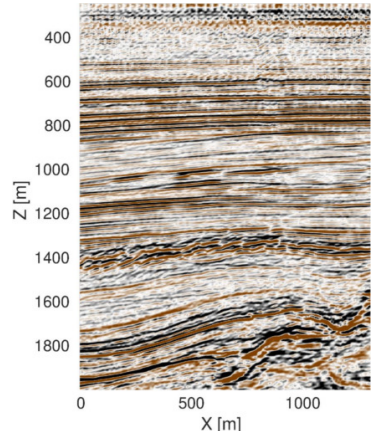

(a)

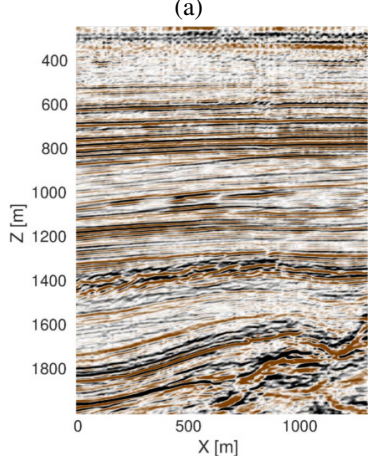

(c)

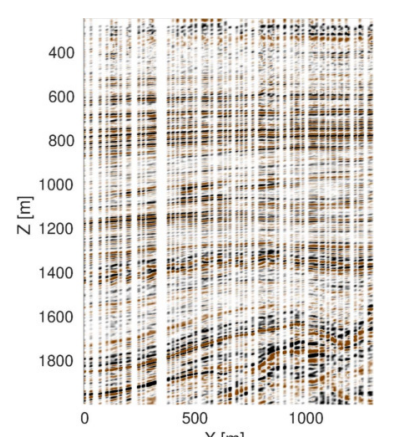

(b)

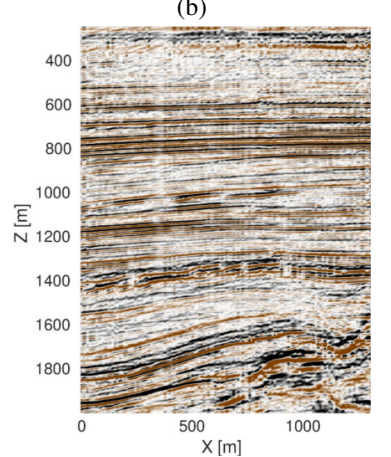

(d)
Fig. 7. Reconstruction example of field data. (a) complete data (b) corrupted data (c) reconstruction of DIP method (d) reconstruction of MSSA method

\section{CONCLUSION}

We proposed an unsupervised CNN-based reconstruction strategy for 3D seismic cubes that does not rely on standard training procedures.

A 3D version of the MultiResUNet acts as a deep prior for constraining the ill-posed interpolation problem. Compared to standard U-Net applied in previous works, this architecture captures inner data features at different scales, and 3D convolutions exploit correlation in both horizontal and vertical directions, making the prior information more effective. Results achieved on both synthetic and field data demonstrate the convincing abilities of the proposed workflow when reconstructing irregularly sampled data even in the challenging case of weak events.

Computational costs are significantly reduced by properly presetting the initial CNN weights. Future studies will continue to address the issues related to computational cost and the reconstruction of aliased events.

\section{REFERENCES}

[1] W. Chang and G. Mcmechan, "3d acoustic prestack reverse-time migration," Geophysical Prospecting, vol. 38, no. 7, pp. 737-755, 2006.

[2] J. Virieux and S. Operto, "An overview of full-waveform inversion in exploration geophysics," Geophysics, vol. 74, no. 6, pp. WCC1-WCC26, 2009.
[3] D. Verschuur, A. Berkhout, and C. Wapenaar, "Adaptive surface-related multiple elimination," Geophysics, vol. 57, no. 9, pp. 1166-1177, 1992.

[4] R. H. Stolt, "Seismic data mapping and reconstruction," Geophysics, vol. 67 , no. 3, pp. 890-908, 2002.

[5] S. Fomel, "Seismic reflection data interpolation with differential offset and shot continuation," Geophysics, vol. 68, no. 2, pp. 733-744, 2003.

[6] S. Spitz, "Seismic trace interpolation in the fx domain," Geophysics, vol. 56, no. 6, pp. 785-794, 1991.

[7] S. R. Trickett, "F-xy eigenimage noise suppression," Geophysics, vol. 68, no. 2, pp. 751-759, 2003.

[8] V. Oropeza and M. Sacchi, "Simultaneous seismic data denoising and reconstruction via multichannel singular spectrum analysis," Geophysics, vol. 76, no. 3, pp. V25-V32, 2011.

[9] A. Adamo, P. Mazzucchelli, and N. Bienati, "Irregular interpolation of seismic data through low-rank tensor approximation," in Geoscience and Remote Sensing Symposium (IGARSS), 2015 IEEE International. IEEE, 2015, pp. 4292-4295.

[10] M. A. Nazari Siahsar, S. Gholtashi, E. Olyaei Torshizi, W. Chen, and $\mathrm{Y}$. Chen, "Simultaneous denoising and interpolation of 3-d seismic data via damped data-driven optimal singular value shrinkage," IEEE Geoscience and Remote Sensing Letters, vol. 14, no. 7, pp. 1086-1090, 2017.

[11] M. Naghizadeh, "Seismic data interpolation and denoising in the frequency-wavenumber domain," Geophysics, vol. 77, no. 2, pp. V71V80, 2012.

[12] B. Wang, "An efficient pocs interpolation method in the frequency-space domain," IEEE Geoscience and Remote Sensing Letters, vol. 13, no. 9, pp. 1384-1387, 2016.

[13] F. J. Herrmann and G. Hennenfent, "Non-parametric seismic data recovery with curvelet frames," Geophysical Journal International, vol. 173, no. 1, pp. 233-248, 2008.

[14] S. Gan, S. Wang, Y. Chen, Y. Zhang, and Z. Jin, "Dealiased seismic data interpolation using seislet transform with low-frequency constraint," IEEE Geoscience and remote sensing letters, vol. 12, no. 10, pp. 21502154,2015

[15] L. Zhu, E. Liu, and J. H. McClellan, "Joint seismic data denoising and interpolation with double-sparsity dictionary learning," Journal of Geophysics and Engineering, vol. 14, no. 4, p. 802, 2017.

[16] S. Mandelli, F. Borra, V. Lipari, P. Bestagini, A. Sarti, and S. Tubaro, "Seismic data interpolation through convolutional autoencoder," in $S E G$ Technical Program Expanded Abstracts, 2018, pp. 4101-4105.

[17] B. Wang, N. Zhang, W. Lu, and J. Wang, "Deep-learning-based seismic data interpolation: A preliminary result," Geophysics, vol. 84, no. 1, pp. V11-V20, 2018

[18] A. Siahkoohi, R. Kumar, and F. Herrmann, "Seismic data reconstruction with generative adversarial networks," in 80th EAGE Conference and Exhibition, 2018.

[19] A. Mikhailiuk and A. Faul, "Deep learning applied to seismic data interpolation," in 80th EAGE Conference and Exhibition, 2018.

[20] D. Oliveira, R. Ferreira, R. Silva, and E. Brazil, "Interpolating seismic data with conditional generative adversarial networks," IEEE Geoscience and Remote Sensing Letters, vol. 15, no. 12, pp. 1952-1956, 2018.

[21] H. Kaur, N. Pham, and S. Fomel, "Seismic data interpolation using cyclegan," in SEG Technical Program Expanded Abstracts, 2019, pp. 2202-2206.

[22] D. Ulyanov, A. Vedaldi, and V. Lempitsky, "Deep image prior," in The IEEE Conference on Computer Vision and Pattern Recognition (CVPR), June 2018.

[23] Q. Liu, L. Fu, and M. Zhang, "Deep-seismic-prior-based reconstruction of seismic data using convolutional neural networks," arXiv preprint arXiv: 1911.08784, 2019.

[24] F. Kong, V. Lipari, P. Bestagini, and S. Tubaro, "A deep prior convolutional autoencoder for seismic data interpolation," in 82nd EAGE Conference and Exhibition, 2020.

[25] O. Ronneberger, P. Fischer, and T. Brox, "U-net: Convolutional networks for biomedical image segmentation," in International Conference on Medical image computing and computer-assisted intervention. Springer, 2015, pp. 234-241.

[26] N. Ibtehaz and M. S. Rahman, "Multiresunet: Rethinking the u-net architecture for multimodal biomedical image segmentation," Neural Networks, vol. 121, pp. 74-87, 2020.

[27] R. M. Silva, L. Baroni, R. S. Ferreira, D. Civitarese, D. Szwarcman, and E. V. Brazil, "Netherlands dataset: A new public dataset for machine learning in seismic interpretation," 2019. 